\title{
Ganhos Potenciais em Esperança de Vida com a Exclusão das Mortes por Neoplasias Malignas em Fortaleza, 1993-95*
} Potential Gains in Life Expectancy with the Exclusion of the Deaths by Cancer in Fortaleza, 1993-95

Marcelo Gurgel Carlos da Silva

\section{RESUMO}

O objetivo do estudo foi avaliar, através das tábuas de vida de múltiplo decremento, a magnitude da atuação dos cânceres nas probabilidades de morte, de sobrevivência e nas esperanças de vida, de acordo com sexo e idade, dos residentes no município de Fortaleza (Brasil), em 1993-95. A esperança de vida ao nascer foi 65,44 anos nos homens, e 73,33 anos nas mulheres. Constatou-se que se as neoplasias malignas não tivessem sido causa de morte haveria um incremento de 1,60 e 1,91 anos, respectivamente, nas expectativas de vida ao nascer, nos sexos masculino e feminino.

Palavras-chave: neoplasias; epidemiologia; mortalidade; tábuas de vida; esperança de vida; Brasil.

\section{ABSTRACT}

The aim of this study was to evaluate, through life tables of multiple decrement, the magnitude of the role of the cancer upon the probabilities of death and survival, and upon life expectancy of the residents of Fortaleza (Brazil), grouped according sex and age, in the period 1993-95. Life expectancy at birth was 65.44 years, for men, and 73.33 years for women. It was established that if cancers had not been the cause of death, there would be an increase of 1.60 and 1.91 years, respectively, in the life expectancy at birth for males and females.

Key words: neoplasms; epidemiology; mortality; life tables; life expectancy; Brazil. 


\section{INTRODUÇÃO}

0 estudo da mortalidade, enfocando as suas causas, é de capital relevância para o diagnóstico de saúde e para identificar as prioridades do setor a serem atendidas. Por isso, torna-se fundamental a utilização de indicadores que possam aferir a magnitude, o impacto e a transcendência das diversas causas, conforme critérios racionais adotados em planejamento em saúde. ${ }^{1}$

Para a avaliação do impacto das doenças no contexto da saúde da população, dentre os indicadores recomendados, o estudo dos anos potenciais de vida perdidos tem merecido o especial cuidado. Esse estudo vem sendo operacionalizado por duas técnicas distintas: a primeira, proposta e desenvolvida por $C$ hiang ${ }^{2}$ envolve a utilização de processos estocásticos, através das tábuas de vida de múltiplo decremento; e a outra, sugerida por Romeder e M cW hinnie, ${ }^{3}$ se fundamenta nos anos de vida restantes em cada grupo etário em relação à idade limite préestabelecida.

Arriaga ${ }^{4}$ apresentou um novo índice de anos de vida perdidos que permite relacionar a mortalidade de determinadas causas de morte com a mudança da esperança de vida ao nascer ou entre idades selecionadas. Esse índice recoloca o uso de tábua de vida de múltiplo decremento para analisar o impacto sobre a esperança de vida que produz a mudança da mortalidade por causa e idade, além de permitir analisar historicamente a mortalidade por causa sem necessidade de usar taxas padronizadas; esse novo índice pode ser utilizado para detectar causas de morte que mudem rapidamente, ainda que o nível de mortalidade seja relativamente baixo.

Bocco, ${ }^{5}$ retomando 0 estudo Arriaga, ${ }^{4}$ apresenta uma nova versão, contínua, do índice dos anos de vida perdidos, diferenciando pelas distintas causas de morte e demonstrando a relação entre esse índice e a esperança de vida temporária nos campos contínuo ediscreto. As vantagens demográficas deste desenvolvimento matemático estão em que ele permite relacionar mais clara e explicitamente as medidas de sobrevivência com as dos anos de vida perdidos.
Com efeito, a construção de tábuas de vidas específicas, que permitam estudar e cotejar a esperança de vida com várias doenças sucessivamente eliminadas, traduz 0 melhor meio para aferir a importância relativa dos vários agravos e saúde para o bem-estar econômico de uma população.

Processos estocásticos têm sido comumente empregados e citados para medir 0 impacto do câncer, a exemplo dos trabalhos de Barnum e Greeberg, ${ }^{6}$ Bonneux et al. ${ }^{7} \mathrm{e}$ $M$ erril et al.; 8 o risco de desenvolvimento da doença, conforme estudos de Fraser e Shavlik, ${ }^{9}$ Karner- $\mathrm{H}$ anusch et al., ${ }^{10} \mathrm{M}$ anton e D owd, ${ }^{11} \mathrm{M}$ erril, ${ }^{12} \mathrm{M}$ erril et al., ${ }^{13} \mathrm{~N}$ azario et al. ${ }^{14}$ e Wun et al., ${ }^{15}$ ou para avaliar intervenções, como os de Agarwal et al.,16 Messori e Trippoli ${ }^{17}$ e Soutter. ${ }^{18}$

Para o Brasil em 2001, o M inistério da Saúde estima que serão registrados 305.330 casos novos ( 150.450 em homens e 154.880 em mulheres) e 117.550 óbitos por câncer (63.330 em homens e 54.220 em mulheres); para Fortaleza em 2001, foram estimadas as taxas de mortalidade de 81,24 por 100.000 homens e 72,75 por 100.000 mulheres e de incidência da ordem de 165,72 por 100.000 homens e 177,52 por 100.000 mulheres. ${ }^{19}$

As neoplasias malignas respondem presentemente por considerável parte das mortes entre os residentes de Fortaleza, sobretudo em seus contingentes populacionais mais idosos. Silva ${ }^{20}$ relata que as neoplasias malignas, em Fortaleza, no período de 197880 , contribuíram com $5,69 \%$ das mortes masculinas e $8,64 \%$ das femininas, significando taxas de 55,18 por 100.000 homens e 58,49 por 100.000 mulheres, sendo que tais cifras cresceram de tal modo que, em 1993-95, passaram a responder por $10,27 \%$ e $14,18 \%$, respectivamente, com coeficientes de 67,34 por 100.000 homens e 62,04 por 100.000 mulheres.

0 objetivo deste trabalho é analisar a magnitude da atuação das neoplasias malignas nas probabilidades de morte, de sobrevivência e nas esperanças de vida, segundo sexo eidade, dos residentes no município de Fortaleza, no período 1993-95, utilizando a metodologia das tábuas de vida de múltiplo decremento. 


\section{MATERIAL E MÉTODOS}

\section{MORTALIDADE}

0 material básico foi obtido a partir da consolidação de resultados do levantamento das declarações de óbitos dos residentes em Fortaleza, em 1993-95, efetuado pela Secretaria de Saúde do Estado do Ceará, e divulgado em CD-ROM do Ministério da Saúde. Foram agrupadas como neoplasias malignas as causas correspondentes aos códigos 140 a 208, conforme a Classificação Internacional de D oenças - Revisão 1975. ${ }^{21}$ $0 \mathrm{~s}$ dados referentes à distribuição dos óbitos, por sexo e idade, para todas as causas e as neoplasias malignas estão expostos na Tabela 1.

\section{POPULAÇÃO}

A população empregada foi a utilizada por Silva, ${ }^{20}$ para Fortaleza em 01.07.94, que correspondia à média do triênio 1993-95. Essa população foi obtida pelo método geométrico, por meio da interpolação dos resultados do Censo de 1991 e da Contagem de 1996, e sua distribuição por sexo e idade tomou por base os valores censitários de 1991 (Tabela 2).

\section{TÁBUAS DE VIDA}

A esperança de vida foi determinada através da tábua de vida constituída de variáveis representadas em colunas a seguir explicitadas:

Coluna (1): $x$ - Intervalo de idade $(x, x+n)$. Esse intervalo é definido entre duas idades exatas, excetuando 0 grupo de 85 e mais anos.

Coluna (2): $1_{x}$ - Número de sobreviventes que iniciam a idade $x .0$ seu valor inicial $\left(1_{0}\right)$ éa raiz cem mil nascimentos vivos e os demais valores correspondem aos sobreviventes em cada idade $x$, obtidos da diferença numérica entre as colunas (2) e (4).

Coluna (3): ${ }_{n} q_{x}$ - Proporção de mortes no intervalo etário $(x, x+n)$. É a coluna mestra para a construção da tábua de vida, dado que as diversas colunas dependem de seus resultados para a determinação de seus valores específicos. Seu cálculo envolve o relacionamento dos óbitos em determinada faixa etária e a correspondente população sob risco.

\begin{tabular}{|c|c|c|c|c|c|c|}
\hline \multirow{3}{*}{ beda } & \multicolumn{2}{|c|}{ Todas es Causes } & \multicolumn{4}{|c|}{ Nesplesios Malignos } \\
\hline & \multirow{2}{*}{$\begin{array}{c}\text { Ussadizo } \\
n^{\circ}\end{array}$} & \multirow{2}{*}{$\begin{array}{c}\text { Feniniro } \\
n^{\circ}\end{array}$} & \multicolumn{2}{|c|}{ Mossulim } & \multicolumn{2}{|c|}{ Feniniro } \\
\hline & & & $n^{\circ}$ & 5 & $n^{\circ}$ & gr \\
\hline 0 & 2369 & 1804 & 1 & 0,04 & 1 & 0,06 \\
\hline 1 & 250 & 233 & 1 & 0,40 & 3 & 1,29 \\
\hline 2 & 84 & 64 & 1 & 1,19 & 1 & 1,56 \\
\hline 3 & 5) & 32 & 4 & 7,84 & 2 & 6,25 \\
\hline 4 & 50 & 32 & 6 & 12,00 & 1 & 3,13 \\
\hline 5.9 & 198 & 122 & 20 & 10,10 & 15 & 1230 \\
\hline 10.14 & 179 & 110 & 16 & 8,94 & 13 & 11,82 \\
\hline $15-19$ & 463 & 183 & 18 & 3,89 & 17 & 9,29 \\
\hline $20-24$ & 680 & 223 & 31 & 4,56 & 23 & 10,31 \\
\hline 25.29 & 786 & 224 & $\eta$ & 3,44 & $x$ & 11,16 \\
\hline $30-34$ & 763 & 259 & 32 & 4,19 & 40 & 15,44 \\
\hline $35-39$ & 342 & 296 & 39 & 5,26 & 61 & 20,81 \\
\hline $40-44$ & 349 & 432 & 59 & 7,88 & 140 & 32,41 \\
\hline $45-49$ & 848 & 526 & 113 & 13,33 & 154 & 29,28 \\
\hline 50.54 & 876 & 588 & 138 & 15,53 & 164 & 2789 \\
\hline $55-59$ & 994 & 709 & 163 & 16,40 & 206 & 29,06 \\
\hline $60-64$ & 1.109 & 840 & 219 & 19,75 & 210 & 25,00 \\
\hline $65-69$ & 1,390 & 1.151 & 262 & 18,99 & 209 & 18,16 \\
\hline 70.74 & 1,336 & 1.178 & 205 & 15,34 & 210 & 17,83 \\
\hline $75-79$ & 1270 & 1.290 & 194 & 15,28 & $1 n$ & 13,33 \\
\hline $80-84$ & 1.128 & 1,328 & 131 & 11,61 & 132 & 9,94 \\
\hline $858+$ & 1.132 & 1833 & 106 & 9,36 & 105 & 573 \\
\hline Istal" & 17437 & 13.457 & 1.784 & 10,23 & 1.904 & 14,15 \\
\hline
\end{tabular}

*exclui óbitos de idade ignorada

\begin{tabular}{c|c|c|}
\hline \multicolumn{3}{|c|}{$\begin{array}{c}\text { Tabela 2 - População estimada de Fortaleza } \\
\text { por sexo e idade, para } 01.07 .94\end{array}$} \\
\hline Idada & Masculino & Feminino \\
\hline 0 & 20.197 & 19.202 \\
1 & 20.889 & 20.047 \\
2 & 23.644 & 22.809 \\
3 & 23.314 & 22.841 \\
4 & 22.845 & 22.144 \\
$5-9$ & 111.871 & 110.848 \\
$10-14$ & 101.248 & 108.385 \\
$15-19$ & 90.847 & 112.765 \\
$20-24$ & 91.998 & 110.118 \\
$25-29$ & 84.463 & 101.041 \\
$30-34$ & 65.497 & 78.663 \\
$35-39$ & 52.876 & 65.839 \\
$40-44$ & 45.072 & 54.647 \\
$45-49$ & 33.159 & 41.152 \\
$50-54$ & 27.941 & 35.525 \\
$55-59$ & 20.728 & 27.248 \\
$60-64$ & 17.242 & 24.230 \\
$65-69$ & 13.770 & 18.874 \\
$70-74$ & 8.114 & 11.725 \\
$75-79$ & 5.601 & 9.034 \\
$80-84$ & 2.032 & 4.055 \\
$85+$ & 2.708 & 4.444 \\
\hline Total & 886.056 & 1.025 .636 \\
\hline
\end{tabular}


Empregou-se a seguinte fórmula:

$$
{ }_{n} q_{x}=\frac{n_{n} 0_{x}}{{ }_{n} p_{x}+\left(1-a^{\prime}\right) n_{n_{n}} 0_{x}} \text {; nde: }
$$

${ }_{n} 0_{x}=$ óbitos de intervalo etário $(x, x+n)$

${ }_{n} p_{x}=$ população do intervalo etário $(x, x+n)$

$a^{\prime}=$ fator de separação

Foram adotados para ( $\left.a^{\prime}\right)$ os valores 0,20 em menores de 1 ano, 0,30 na idade de um ano e 0,50 nas demais idades nos sexos masculino e feminino.

$0{ }_{n} 0_{x}$ nos diversos grupos é multiplicado pelo número de anos do respectivo intervalo. $\mathrm{N}$ a tábua, ${ }_{n} q_{x}$ é multiplicado por 1000.

Coluna (4): ${ }_{n} d_{x}$ - Número de mortes no intervalo $(x, x+n)$. Seus valores são oriundos da multiplicação dos ${ }_{n} q_{x}$ pelos sobreviventes à mesma idade e traduzem o número de óbitos da tábua da vida.

Coluna (5): ${ }_{n} \mathrm{~L}_{x}$ - Número de anos vividoso intervalo $(x, x+n)$. Cada indivíduo sobrevivente do ano contribui com um ano e cada óbito com a fração do ano ( $\left.a^{\prime}\right)$, de modo que os valores de ${ }_{n} L_{x}$ provem dessa adição. Assim, $O_{n} L_{x}$ a idade de um ano representa a soma dos óbitos dessa idade multiplicado pelo respectivo fator de separação $(0,3)$ com o total de sobreviventes assinalado à idade de dois anos.

Para 0 grupo de 85 e mais anos, $O_{n} L_{x}$ foi determinado pela fórmula recomendada por Barclay referida por Laurenti et al. ${ }^{22}$

Coluna (6): $T_{x}$ - Total de anos vividos além da idade $x$. Seus resultados obtêm-se da acumulação dos valores expressos na coluna 5, a partir do grupo 85 e mais anos e correspondem à soma do número de anos vividos em cada intervalo de idade.

Coluna (7): $e_{x}$ - Esperança de vida observada na idade $x$. Corresponde ao número médio de anos de vida esperados pelas pessoas na idade $x$. Seus valores resultam da divisão numérica dos dados da coluna (6) pelo da coluna (2).

As Tabelas 2 e 3 mostram as tábuas de sobrevivência de Fortaleza, em 1993-95, respectivamente, para as populações masculina e feminina.

TÁBUAS DE VIDA DE MÚLTIPLO DECREMENTO

A elaboração dasT ábuas deVida de Múltiplo D ecremento (TVM D) seguiu a metodologia de C hiang. ${ }^{2}$ Sua construção diverge daquela da tábua de vida somente pela coluna 3 , em que a probabilidade de morte é líquida $\left(q_{x}\right)$, ou seja, aceita que uma determinada causa ou conjunto de causas tenham sido excluídos da população.
A probabilidade líquida de morte $\left(q_{x}\right)$ é estimada por:

$q_{x .}=1-p_{i}\left[\left(D_{i}-\pi_{i j} D_{i j}\right) / D_{j}\right]$

em que $p_{i}=$ estimador da probabilidade de um indivíduo sobreviver de $x_{i} a x_{i+n}$ e é igual $a:$

$$
\begin{aligned}
& \mathrm{p}_{\mathrm{i}}=1 \mathrm{a}_{\mathrm{i}}^{\prime} \mathrm{N}_{i} \mathrm{M}_{\mathrm{i}} \text {; } \text {; onde: } \\
& 1+\left(1-a_{i}^{\prime}\right) N_{i} M_{i} \\
& \mathrm{I}=0,1,2
\end{aligned}
$$
múltiplo decremento seriam hipotéticas, em decorrência de se fundamentarem na suposição da inexistência de determinada enfermidade como causa de morte, pode ser empregada, conforme Gotlieb, ${ }^{23}$ a nomenclatura de probabilidade de morte hipotética $\left(q_{x}\right)$, probabilidade de sobreviver hipotética $\left(P_{0 x}\right)$ e esperança de vida hipotética $\left(e_{x}\right)$ para as suas variáveis; as correspondentes variáveis encontradas na tábua de vida são ditas reais e representadas por: $q_{x,} P_{0 x}$ e $e_{x}$.

As $P_{0 x}$ podem ser obtidas da divisão de $1_{x}$ por $1_{0}$ e retratam a probabilidade de sobreviver do nascimento até a idade $x$.

Por intermédio da comparação do número médio de anos vividos entre 15 e 65 anos usando a fórmula $T_{15}-T_{65} / 1_{15}$, com as sucessivas eliminações de causa podem ser também identificados os ganhos potenciais de vida ativa.

\section{RESULTADOS}

\section{ESPERANÇA DE VIDA}

Entre os homens, a esperança de vida ao nascer foi de 65,44 anos e, ao primeiro ano, de 67,02 anos, revelando um aumento de 1,58 anos e incremento relativo de $2,41 \%$ se sobreviver ao primeiro ano de vida. No sexo feminino, os valores encontrados foram de 73,33 e 74,64 anos, respectivamente, para a esperança de vida ao nascer e à idade de um ano, portanto, uma diferença de 1,31 anos e acréscimo de 1,79\% (Tabelas 3 e 4). 


\begin{tabular}{|c|c|c|c|c|c|c|}
\hline $\begin{array}{c}x \\
\text { (1) }\end{array}$ & $\begin{array}{l}1_{x} \\
(2)\end{array}$ & $\begin{array}{c}1.000 n q_{x} \\
\text { (3) }\end{array}$ & $\begin{array}{l}n^{d} d_{x} \\
\text { (4) }\end{array}$ & $\begin{array}{l}n L_{n} \\
\text { (5) }\end{array}$ & $\begin{array}{l}T_{x} \\
{[6]}\end{array}$ & $\begin{array}{l}e_{x} \\
\langle 7\rangle\end{array}$ \\
\hline 0 & 100.000 & 37,92 & 3.792 & 96,967 & 6.544 .349 & 65,44 \\
\hline 1 & 96.208 & 3,98 & 384 & 95.940 & $6.447,382$ & 67,02 \\
\hline 2 & 95.824 & 1,19 & 114 & 95.767 & 6.351 .442 & 66,28 \\
\hline 3 & 95.710 & 0,73 & 71 & 95.675 & 6.255 .675 & 65,36 \\
\hline 4 & 95.639 & 0,73 & 71 & 95.604 & 6.160 .000 & 64,41 \\
\hline $5-9$ & 95.568 & 2,95 & 282 & 477.135 & 6.064 .396 & 63,46 \\
\hline 10.14 & 95.286 & 2,95 & 281 & 475.726 & 5.587 .261 & 58,64 \\
\hline $15=19$ & 95.004 & 8,46 & 805 & 473.011 & 5.111 .535 & 53,80 \\
\hline $20-24$ & 94.200 & 12,25 & 1.154 & 468.114 & 4.638 .524 & 49.24 \\
\hline $25-29$ & 93.046 & 15,40 & 1.433 & 461.646 & $4.170,410$ & 44,82 \\
\hline $30-34$ & 91.613 & 19,23 & 1.763 & 453.657 & 3.708 .764 & 40,48 \\
\hline $35-39$ & 89.850 & 23,12 & 2.078 & 444.055 & 3.255 .108 & 36,23 \\
\hline $40-44$ & 87.772 & 27,32 & 2.399 & 432.863 & 2.811 .052 & 32,03 \\
\hline $45-49$ & 85.373 & 41,74 & 3.564 & 417.957 & 2.378 .189 & 27,86 \\
\hline 50.54 & 81.809 & 50,93 & 4.167 & 398.630 & 1.960 .232 & 23,96 \\
\hline 55.59 & 77.642 & 76,86 & 5.968 & 373.293 & 1.561 .602 & 20,11 \\
\hline $60-64$ & 71.675 & 101,75 & 7.293 & 340,140 & 1.188 .309 & 16,58 \\
\hline $65-69$ & 64.381 & 154,16 & 9.926 & 297.092 & 848.169 & 13,17 \\
\hline $70-74$ & 54.456 & 241,32 & 13.142 & 239.425 & 551.077 & 10,12 \\
\hline $75-79$ & 41.314 & 317,85 & 13.132 & 173.740 & 311.653 & 7,54 \\
\hline $80-84$ & 28.182 & 632,58 & 17.828 & 96.340 & 137.913 & 4,89 \\
\hline $85+$ & 10.354 & 100,00 & 10.355 & 41.573 & 41.573 & 4,02 \\
\hline
\end{tabular}

\begin{tabular}{|c|c|c|c|c|c|c|}
\hline $\begin{array}{c}x \\
\text { (1) }\end{array}$ & $\begin{array}{l}1_{x} \\
|2\rangle\end{array}$ & $\begin{array}{c}1.000{ }_{n} q_{x} \\
\text { (3) }\end{array}$ & $\begin{array}{l}n^{d_{x}} \\
\text { (4) }\end{array}$ & $\begin{array}{l}{ }_{n} L_{1} \\
(5)\end{array}$ & $\begin{array}{l}\mathrm{T}_{\mathrm{n}} \\
(6)\end{array}$ & $\begin{array}{l}a_{x} \\
\langle 7\rangle\end{array}$ \\
\hline 0 & 100.000 & 30,56 & 3.056 & 97.556 & 7.332 .981 & 73,33 \\
\hline 1 & 96.944 & 3,87 & 376 & 96.682 & 7.235 .425 & 74,64 \\
\hline 2 & 96.568 & 0,94 & 91 & 96.523 & 6.713 .515 & 73,92 \\
\hline 3 & 96.477 & 0,47 & 46 & 96.455 & 6.622 .239 & 72,99 \\
\hline 4 & 96,431 & 0,49 & 47 & 96.408 & 6.531 .190 & 72,03 \\
\hline 5.9 & 96,384 & 1,84 & 178 & 481.475 & 6.440 .268 & 71,06 \\
\hline $10-14$ & 96.206 & 1,70 & 164 & 480.622 & 5.986 .633 & 66,19 \\
\hline $15-19$ & 96.042 & 2,71 & 260 & 479.562 & 5.534 .248 & 61,30 \\
\hline $20-24$ & 95.782 & 3,37 & 324 & 478.102 & 5.083 .026 & 56,46 \\
\hline $25-29$ & 95.458 & 3,69 & 353 & 476.410 & 4.633 .466 & 51,64 \\
\hline $30-34$ & 95.105 & 5,48 & 521 & 474.223 & 4.185 .796 & 46,82 \\
\hline 35.39 & 94.584 & 7,47 & 707 & 471.152 & 3.740 .546 & 42,07 \\
\hline $40-44$ & 93.877 & 13,09 & 1.230 & 466.310 & 3.298 .829 & 37,37 \\
\hline 45.49 & 92.647 & 21,08 & 1.954 & 458.351 & 2.861 .817 & 32,83 \\
\hline $50-54$ & 90.693 & 27,22 & 2.469 & 447.295 & 2.431 .703 & 28,48 \\
\hline 55.59 & 88.224 & 42,45 & 3.746 & 431.758 & 2.011 .142 & 24,21 \\
\hline $60-64$ & 84.479 & 56,16 & 4.745 & 410.531 & 1.604 .375 & 20,17 \\
\hline $65-69$ & 79.734 & 96,73 & 7.713 & 379.386 & 1.216 .301 & 16,22 \\
\hline 70.74 & 72.021 & 154,52 & 11.129 & 332.281 & 851.911 & 12,69 \\
\hline 75.79 & 60.892 & 212,69 & 12.951 & 272.081 & 526.641 & 9,56 \\
\hline $80-84$ & 47.940 & 428,81 & 20.558 & 188.308 & 257.321 & 6,46 \\
\hline $85+$ & 27.383 & 100,00 & 27.383 & 121.510 & 68.917 & 4,44 \\
\hline
\end{tabular}

\section{TÁBUAS DE VIDA DE MÚLTIPLO DECREMENTO}

Analisando a atuação das neoplasias malignas nas probabilidades de morte segundo sexo e grupo etário, nota-se que, em menores de um ano, elas têm importância mínima, pois a exclusão dos cânceres como causa de morte significaria uma redução de probabilidades de morte da ordem de 0,00002 e 0,00002, para os sexos masculino e feminino, respectivamente, correspondendo às diferenças relativas de $0,05 \%$ e $0,06 \%$. Em que pesem as variações nos menores de 15 anos, possivelmente explicáveis pelo reduzido número de óbitos por câncer na infância, nas idades subseqüentes, as diferenças relativas das probabilidades de morte real $\mathrm{e}$ hipotética crescem progressivamente, atingindo um nível máximo, na faixa de 60-64 anos 
$(18,91 \%)$, nos homens e em $40-44$ anos $(32,24 \%)$, nas mulheres, e depois as cifras declinam. Valores relativos superiores a $15 \%$ nas probabilidades de morrer foram vistos nos grupos etários compreendidos entre 50 a 69 anos nos homens e entre 35 a 74 anos nas mulheres. As diferenças relativas no sexo feminino são, de modo geral, mais altas do que as do masculino (Tabelas 3-7).

As probabilidades de sobreviver, real e hipotética, pela eliminação dos cânceres como causa de morte são apresentadas na Tabela 8, onde se constata que essas probabilidades, aos 85 anos e mais, passam de 0,10354 para 0,14024 , no sexo masculino, e de 0,27383 para 0,32952, nas mulheres. Isto, em relação à tábua de vida, significa que dos 100.000 nascidos vivos de raiz $1_{0}$, acatando a premissa da exclusão dos cânceres, chegarão aos 85 anos $35,45 \%$ a mais entre os homens (de 10.354 para 14.024 homens) e $20,34 \%$ a mais nas mulheres (de 27.383 para 32.952 mulheres).
Considerando que as neoplasias malignas atingem predominantemente contingentes de adultos, torna-se interessante avaliar o impacto das causas externas entre a população economicamente ativa (15 a 64 anos), ou seja, dado que o indivíduo tenha chegado aos 15 anos, qual a sua probabilidade de atingir aos 65 anos. Assim, verificou-se, na população citada, que os valores hipotéticos seriam de $71,51 \%$ e $87,16 \%$ para os sexos masculino e feminino, respectivamente, em contraste aos dados reais de $67,77 \%$ e $83,02 \%$, revelando incrementos relativos de $5,52 \%$ e $4,99 \%$.

As probabilidades de sobreviver, real e hipotética, estão representadas nas Figuras 1 e 2 e Tabela 8, onde verifica-se que 0 sexo feminino seria melhor contemplado com a exclusão das neoplasias malignas até aos 64 anos, ficando a partir dos 65 anos a predominância para 0 masculino.

\begin{tabular}{|c|c|c|c|c|c|c|}
\hline $\begin{array}{c}x \\
{[1]}\end{array}$ & $\begin{array}{l}1 x \\
(2)\end{array}$ & $\begin{array}{c}1.000 n 4 x \\
\text { (3) }\end{array}$ & $\begin{array}{l}n^{n} d_{n} \\
{[4]}\end{array}$ & $\begin{array}{l}n L_{x} \\
(5)\end{array}$ & $\begin{array}{l}\mathrm{T}_{\mathrm{x}} \\
{[6]}\end{array}$ & $\begin{array}{l}e_{x} \\
(7)\end{array}$ \\
\hline 0 & 100.000 & 37,90 & 3.791 & 96.968 & 6.703 .946 & 65,44 \\
\hline 1 & 96.209 & 3,97 & 382 & 95.942 & 6.606 .978 & 67,02 \\
\hline 2 & 95.827 & 1,17 & 113 & 95.771 & 6.511 .036 & 66,28 \\
\hline 3 & 95.714 & 0,68 & 65 & 95.682 & 6.415 .265 & 65,36 \\
\hline 4 & 95.649 & 0,65 & 65 & 95.618 & 6.319 .583 & 64,41 \\
\hline 5.9 & 95.586 & 2,65 & 254 & 477.297 & 6.223 .965 & 63,46 \\
\hline $10-14$ & 95.332 & 2,68 & 256 & 476.021 & 5.746 .667 & 58,64 \\
\hline $15-19$ & 95.076 & 8,14 & 774 & 473.445 & 5.270 .646 & 53,80 \\
\hline $20-24$ & 94.302 & 11,69 & 1.103 & 468.752 & 4.797 .201 & 49,24 \\
\hline $25 \cdot 29$ & 93.199 & 14,87 & 1.386 & 462.528 & 4.328 .450 & 44,82 \\
\hline 30.34 & 91.812 & 18,43 & 1.693 & 454.829 & 3.865 .922 & 40,48 \\
\hline $35-39$ & 90.119 & 21,92 & 1.976 & 445.656 & 3.411 .093 & 36,23 \\
\hline $40-44$ & 88.143 & 25,20 & 2.222 & 435.162 & 2.965 .437 & 32,03 \\
\hline 45.49 & 85.922 & 36,28 & 3.118 & 421.814 & 2.530 .274 & 27,86 \\
\hline $50-54$ & 82.804 & 43,20 & 3.577 & 405.077 & 2.108 .460 & 23,96 \\
\hline $55-59$ & 79.227 & 64,67 & 5.124 & 383.323 & 1.703 .384 & 20,11 \\
\hline $60-64$ & 74.102 & 82,51 & 6.115 & 355.225 & 1.320 .061 & 16,58 \\
\hline $65-69$ & 67.987 & 126,84 & 8.624 & 318.377 & 964.836 & 13,17 \\
\hline 70.74 & 59.363 & 208,48 & 12.376 & 265.875 & 646.459 & 10,12 \\
\hline 75.79 & 46.987 & 276,81 & 13.007 & 202.417 & 380.584 & 7,54 \\
\hline $80-84$ & 33.980 & 587,27 & 19.956 & 120.011 & 178.167 & 4,89 \\
\hline $85+$ & 14.024 & 100,00 & 14.025 & 58.156 & 58.156 & 4,02 \\
\hline
\end{tabular}


Ganhos potenciais em esperança de vida com a exclusão das mortes por neoplasias malignas em Fortaleza, 1993-95

\begin{tabular}{|c|c|c|c|c|c|c|c|c|}
\hline \multicolumn{9}{|c|}{$\begin{array}{l}\text { Tabela } 6 \text { - Tábua de vida de múltiplo decremento, eliminando as neoplasias malignas (C.I.D. 140-208) } \\
\text { como causa de morte, para os residentes no município de Fortaleza, no período 1993-95 (sexo feminino) }\end{array}$} \\
\hline $\begin{array}{c}x \\
\text { (1) }\end{array}$ & \multicolumn{2}{|c|}{$\begin{array}{l}1_{x} \\
(2)\end{array}$} & $\begin{array}{l}500 n 9 x \\
\text { (3) }\end{array}$ & $\begin{array}{l}{ }_{n} d_{x} \\
(4)\end{array}$ & $\begin{array}{l}{ }_{n} L_{x} \\
(5)\end{array}$ & \multicolumn{2}{|c|}{$\begin{array}{c}T_{x} \\
(6)\end{array}$} & $\begin{array}{l}e_{x} \\
(7)\end{array}$ \\
\hline 0 & \multicolumn{2}{|c|}{100.000} & 30,54 & 3.054 & 97.557 & \multicolumn{2}{|c|}{7.524 .380} & 73,33 \\
\hline 1 & \multicolumn{2}{|c|}{96.946} & 3,82 & 371 & 96.687 & \multicolumn{2}{|c|}{7.426 .823} & 74,64 \\
\hline 2 & 96.575 & & 0,93 & 90 & 96.530 & \multicolumn{2}{|c|}{7.330 .136} & 73,92 \\
\hline 3 & 96.485 & & 0,44 & 43 & 96.464 & \multicolumn{2}{|c|}{7.233 .606} & 72,99 \\
\hline 4 & 96.442 & & 0,47 & 46 & 96.419 & \multicolumn{2}{|c|}{7.137 .142} & 72,03 \\
\hline $5-9$ & 96.396 & & 1,61 & 156 & 481.590 & 7.040 & .723 & 71,06 \\
\hline $10-14$ & 96.24 & & 1,50 & 144 & 480.839 & 6.559 & .133 & 66,19 \\
\hline $15-19$ & 96.09 & & 2,46 & 236 & 479.887 & 6.078 & .294 & 61,30 \\
\hline $20-24$ & 95.85 & & 3,03 & 291 & 478.569 & 5.598 & 408 & 56,46 \\
\hline $25-29$ & 95.56 & & 3,28 & 314 & 477.056 & 5.119 & .839 & 51,64 \\
\hline $30-34$ & 95.25 & & 4,63 & 442 & 475.166 & 4.642 & .783 & 46,82 \\
\hline $35-39$ & 94.81 & & 5,94 & 563 & 472.653 & 4.167 & .617 & 42,07 \\
\hline $40-44$ & 94.24 & & 8,87 & 837 & 469.153 & 3.694 & .964 & 37,37 \\
\hline $45-49$ & 93.41 & & 14,96 & 1.398 & 463.567 & 3.225 & .811 & 32,83 \\
\hline $50-54$ & 92.01 & & 19,70 & 1.813 & 455.539 & 2.762 & 44 & 28,48 \\
\hline $55-59$ & 90.20 & & 30,31 & 2.734 & 444.170 & 2.306 & 05 & 24,21 \\
\hline $60-64$ & 87.46 & & 42,43 & 3.711 & 428.056 & 1.862 & .535 & 20,17 \\
\hline $65-69$ & 83.75 & & 79,89 & 6.692 & 402.049 & 1.434 & 79 & 16,22 \\
\hline $70-74$ & 77.06 & & 28,84 & 9.9 & 360. & 1.032 & 30 & 12,69 \\
\hline $75-79$ & 67.13 & & 87,18 & 12.567 & 304. & 671 & & 9,56 \\
\hline $80-84$ & 54.56 & & 396,11 & 21.615 & 218.801 & 367 & 77 & 6,46 \\
\hline $85+$ & 32.95 & & 00,00 & 32.953 & 148.876 & 148 & 876 & 4,44 \\
\hline $\begin{array}{r}\text { Tabela } \\
\text { neop }\end{array}$ & 1. & & & d & & $d / 8$ & . & $\begin{array}{l}\text { ão das } \\
3-95\end{array}$ \\
\hline Sexo & & Mas & sculino & & & Femir & ino & \\
\hline $\begin{array}{c}\text { Idade } \\
\text { (em anos) }\end{array}$ & $q x$ & $9 x$. & $\left|q_{x}-q_{x}\right|$ & $\begin{array}{c}\left|q_{x}-q_{x} \cdot\right| \\
q_{x}(\%)\end{array}$ & $q_{x}$ & $q_{x}$ & $\left|q_{x}=q_{x} \cdot\right|$ & $\begin{array}{c}\left|q_{x}-q_{x}\right| \\
q_{x}(\%)\end{array}$ \\
\hline 0 & 0,03792 & 0,03790 & 0,00002 & 0,05 & 0,03056 & 0,03054 & 0,00002 & 0,06 \\
\hline 1 & 0,00398 & 0,00397 & 0,00001 & 0,25 & 0,00387 & 0,00382 & 0,00005 & 1,29 \\
\hline 2 & 0,00119 & 0,00117 & 0,00002 & 1,68 & 0,00094 & 0,00093 & 0,00001 & 1,06 \\
\hline 3 & 0,00073 & 0,00068 & 0,00005 & 7,35 & 0,00047 & 0,00044 & 0,00003 & 6,38 \\
\hline 4 & 0,00073 & 0,00065 & 0,00008 & 10,96 & 0,00049 & 0,00047 & 0,00002 & 4,08 \\
\hline $5-9$ & 0,00295 & 0,00265 & 0,00030 & 10,17 & 0,00184 & 0,00161 & 0,00023 & 12,50 \\
\hline $10-14$ & 0,00295 & 0,00268 & 0,00027 & 9,15 & 0,00170 & 0,00150 & 0,00020 & 11,76 \\
\hline $15-19$ & 0,00846 & 0,00814 & 0,00032 & 3,78 & 0,00271 & 0,00246 & 0,00025 & 9,23 \\
\hline $20-24$ & 0,01225 & 0,01169 & 0,00056 & 4,57 & 0,00337 & 0,00303 & 0,00034 & 10,09 \\
\hline $25-29$ & 0,01540 & 0,01487 & 0,00053 & 3,44 & 0,00369 & 0,00328 & 0,00041 & 11,11 \\
\hline $30-34$ & 0,01923 & 0,01843 & 0,00080 & 4,16 & 0,00548 & 0,00463 & 0,00085 & 15,51 \\
\hline $35-39$ & 0,02312 & 0,02192 & 0,00120 & 5,19 & 0,00747 & 0,00594 & 0,00153 & 20,48 \\
\hline $40-44$ & 0,02732 & 0,02520 & 0,00212 & 7,75 & 0,01309 & 0,00887 & 0,00422 & 32,24 \\
\hline $45-49$ & 0,04174 & 0,03628 & 0,00546 & 13,08 & 0,02108 & 0,01496 & 0,00612 & 29,03 \\
\hline $50-54$ & 0,05093 & 0,04320 & 0,00773 & 15,18 & 0,02722 & 0,01970 & 0,00752 & 27,63 \\
\hline $55-59$ & 0,07686 & 0,06467 & 0,01219 & 15,86 & 0,04245 & 0,03031 & 0,01214 & 28,60 \\
\hline $60-64$ & 0,10175 & 0,08251 & 0,01924 & 18,91 & 0,05616 & 0,04243 & 0,01373 & 24,45 \\
\hline $65-69$ & 0,15416 & 0,12684 & 0,02732 & 17,72 & 0,09673 & 0,07989 & 0,01684 & 17,41 \\
\hline $70-74$ & 0,24132 & 0,20848 & 0,03284 & 13,61 & 0,15452 & 0,12884 & 0,02568 & 16,62 \\
\hline $75-79$ & 0,31785 & 0,27681 & 0,04104 & 12,91 & 0,21269 & 0,18718 & 0,02551 & 12,00 \\
\hline $80-84$ & 0,63258 & 0,58727 & 0,04531 & 7,16 & 0,42881 & 0,39611 & 0,03270 & 7,63 \\
\hline $85+$ & 1,00000 & 1,00000 & $=$ & - & 1,00000 & 1,00000 & - & - \\
\hline
\end{tabular}


As esperanças de vida ao nascer, com a exclusão das neoplasias malignas, ficaram em 67,04 e 75,24 anos nos sexos masculino e feminino, respectivamente, registrando ganhos potenciais de vida de 1,60 e 1,91 anos; ao primero ano, as esperanças passaram para 68,67 e 76,61 anos, configurando ganhos potenciais de 1,66 e 1,97 anos de vida. Q uanto às diferenças relativas entre as expectativas de vida real e hipotética, têm-se sempre valores mais altos nas mulheres, até aos 50-54 anos, enquanto nos homens nas faixas etárias compreendidas acima dos 55 anos os ganhos relativos se mostram maiores que os verificados nas mulheres; diferenças relativas superiores a $5 \%$ etão qualificadas nos intervalos de 40 a 84 anos no sexo masculino e de 45 a 74 anos no feminino. (Tabelas 3-6 e 9)
As esperanças de vida ativa (15 a 64 anos) com a exclusão das neoplasias malignas ficaram em 45,29 e 48,33 anos nos sexos masculino e feminino, respectivamente, registrando ganhos potenciais de vida ativa de $0,41(0,91 \%)$ e 0,50 anos $(1,05 \%)$ ao se compararem com os níveis originais de 44,88 e 47,83 anos de vida ativa sem a eliminação dessas causas, correspondentemente.

Os ganhos absolutos e relativos em anos de vida com a exclusão dos cânceres como causa de morte são apresentados na Tabela 9 e nas Figuras 3 e 4, onde podem ser verificadas as alterações nas curvas de esperança de vida com a exclusão desses agravos, sobretudo, com maior evidência, entre os homens.

Figura 1 - Probabilidades de sobreviver real $\left(P_{o x}\right)$ e hipotética $\left(P_{o x}{ }\right)$, com eliminação das neoplasias malignas (C.I.D. 140-208) como causa de morte, segundo idade, dos residentes no município de Fortaleza, no período 1996-98 (sexo masculino)

\begin{tabular}{c|cc}
\hline Sexo & $P_{\text {so }}$ & $P_{\text {cos. }}$ \\
\hline 0 & 1,00000 & 1,00000 \\
5 & 0,95568 & 0,95586 \\
10 & 0,95286 & 0,95332 \\
15 & 0,95004 & 0,95076 \\
20 & 0,94200 & 0,94302 \\
25 & 0,93046 & 0,93199 \\
30 & 0,91613 & 0,91812 \\
35 & 0,89850 & 0,90119 \\
40 & 0,87772 & 0,88143 \\
45 & 0,85373 & 0,85922 \\
50 & 0,81809 & 0,82804 \\
55 & 0,77642 & 0,79227 \\
60 & 0,71675 & 0,74102 \\
65 & 0,64381 & 0,67987 \\
70 & 0,54456 & 0,59363 \\
75 & 0,41314 & 0,46987 \\
80 & 0,28182 & 0,33980 \\
$85+$ & 0,10354 & 0,14024 \\
\hline
\end{tabular}

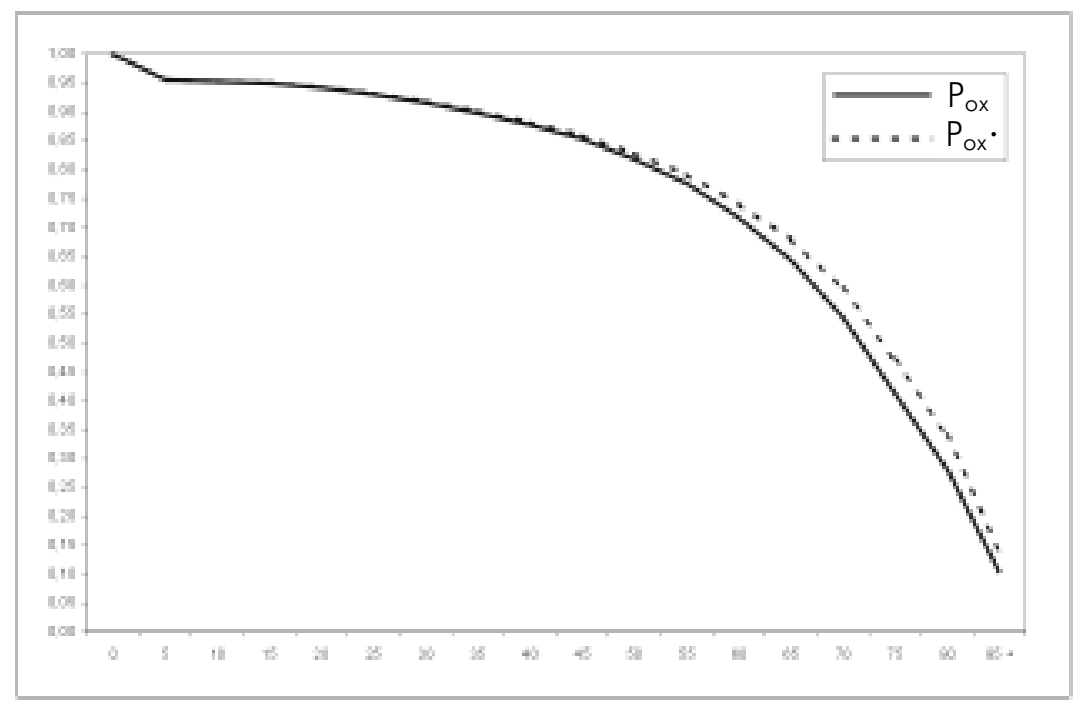

Figura 2 - Probabiblidades de sobreviver real $\left(P_{o x}\right)$ e hipotética $\left(P_{\alpha \times}\right)$, com eliminação das neoplasias malignas (C.I.D. 140-208) como causa de morte, segundo idade, dos residentes de Fortaleza, no período 1996-98 (sexo feminino)

\begin{tabular}{|c|cc|}
\hline Sexo & $P_{\text {ou }}$ & $P_{\text {ce. }}$ \\
\hline 0 & 1,00000 & 1,00000 \\
5 & 0,96384 & 0,96396 \\
10 & 0,96206 & 0,96240 \\
15 & 0,96042 & 0,96095 \\
20 & 0,95782 & 0,95859 \\
25 & 0,95458 & 0,95568 \\
30 & 0,95105 & 0,95254 \\
35 & 0,94584 & 0,94812 \\
40 & 0,93877 & 0,94249 \\
45 & 0,92647 & 0,93412 \\
50 & 0,90693 & 0,92014 \\
55 & 0,88224 & 0,90201 \\
60 & 0,84479 & 0,87467 \\
65 & 0,79734 & 0,83755 \\
70 & 0,72021 & 0,77064 \\
75 & 0,60892 & 0,67135 \\
80 & 0,47940 & 0,54568 \\
$85+$ & 0,27383 & 0,32952 \\
\hline
\end{tabular}

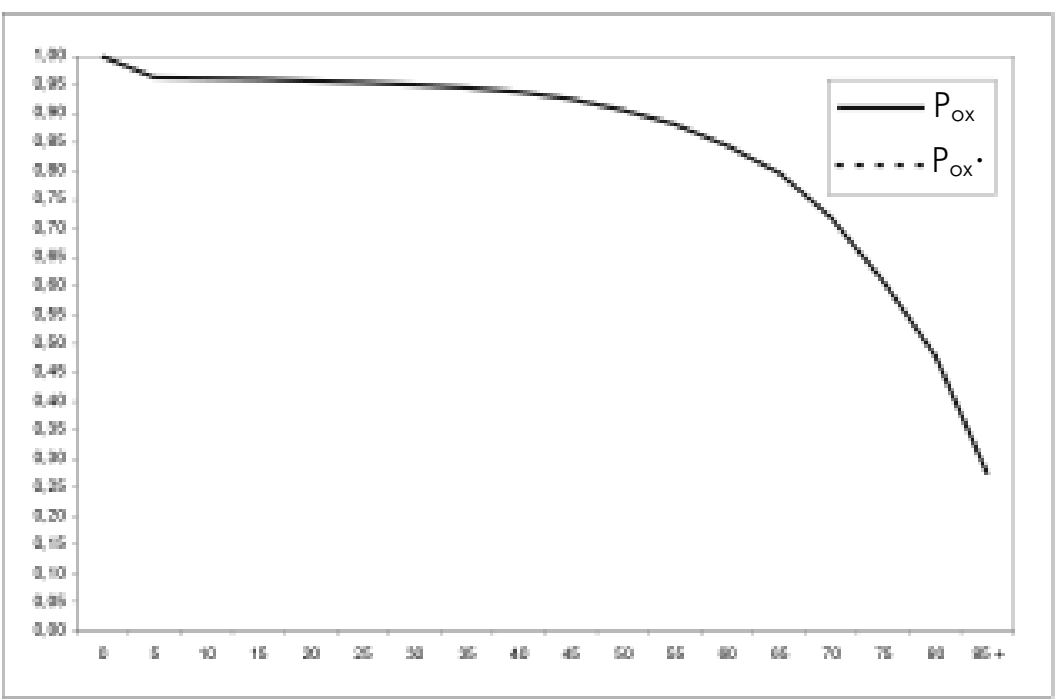




\section{DISCUSSÃO}

A esperança de vida é, sem embargo, um indicador de saúde de alta precisão ea obtenção desta em caráter hipotético, presumindo a exclusão de determinada enfermidade ou lesão como causa de morte, significa um instrumento de grande valor para o planejamento em saúde, em que pesem as dificuldades operacionais da metodologia das tábuas de vida de múltiplo decremento. Vale dizer que a degradação de condições econômicas e sanitárias tem reflexo sucedâneo no declínio da expectativa de vida, a exemplo do observado recentemente na Rússia. ${ }^{24}$

O s cânceres representam importante causa de óbito em Fortaleza, notadamente no sexo feminino, onde, praticamente, de cada sete mortes nesse sexo uma foi por neoplasia, ao passo que essa relação foi uma para dez nos homens (Tabela 1). Comparando com os dados de Fortaleza (1978-80), ganhos em expectativa de vida ao nascer de 1,41 anos (2,38\%), nos homens, e de 2,05 anos $(3,11 \%)$, nas mulheres, constatava-se que Fortaleza detinha riscos mais altos nos homens e mais baixos nas mulheres do que a capital paulista $(20,23)$.

Gotlieb, ${ }^{23}$ em importante estudo original da mortalidade diferencial por causas, analisou várias causas e grupos de causas, dentre os quais os cânceres, para São Paulo, em 1970. Como existe identidade na técnica deste trabalho com 0 de $\mathrm{G}$ otlieb, ${ }^{23}$ alguns de seus achados podem ser cotejados com os de São Paulo, devendo ser ressalvada a diferença temporal de mais de 25 anos, quando São Paulo tinha mais baixa expectativa de vida, o que explica suas mais amplas diferenças relativas.

\begin{tabular}{|c|c|c|c|c|c|c|c|c|}
\hline Sexo & \multicolumn{4}{|c|}{ Masculino } & \multicolumn{4}{|c|}{ Feminino } \\
\hline Idode & P & $P$ & $\mid \mathbf{P}_{\alpha}-\mathbf{P}_{\mathrm{os}}$ & $P_{\text {ex }}-P_{\text {of }}$ & P & P. & $\left|P_{-}-P_{-}\right|$ & $P_{\text {og }}-P_{\text {ox. }}$. \\
\hline [em ancs] & & & & $P_{\text {ox }}$ (⿻) & & & & $P_{\alpha x} / 9$ \\
\hline 0 & 1,00000 & 1,00000 & - & - & 1,00000 & 1,00000 & - & - \\
\hline 1 & 0,96208 & 0,96209 & 1 & 0,00 & 0,96944 & 0,96946 & 2 & 0,00 \\
\hline 2 & 0,95824 & 0,95827 & 3 & 0,00 & 0,96568 & 0,96575 & 7 & 0,01 \\
\hline 3 & 0,95710 & 0,95714 & 4 & 0,00 & 0,96477 & 0,96485 & 8 & 0,01 \\
\hline 4 & 0,95639 & 0,95649 & 10 & 0,01 & 0,96431 & 0,96442 & 11 & 0,01 \\
\hline $5-9$ & 0,95568 & 0,95586 & 18 & 0,02 & 0,96384 & 0,96396 & 12 & 0,01 \\
\hline 10.14 & 0,95286 & 0,95332 & 46 & 0,05 & 0,96206 & 0,96240 & 34 & 0,04 \\
\hline $15-19$ & 0,95004 & 0,95076 & 72 & 0,08 & 0,96042 & 0,96095 & 53 & 0,06 \\
\hline $20-24$ & 0,94200 & 0,94302 & 102 & 0,11 & 0,95782 & 0,95859 & 77 & 0,08 \\
\hline $25 \cdot 29$ & 0,93046 & 0,93199 & 153 & 0,16 & 0,95458 & 0,95568 & 110 & 0,12 \\
\hline $30-34$ & 0,91613 & 0,91812 & 199 & 0,22 & 0,95105 & 0,95254 & 149 & 0,16 \\
\hline 35.39 & 0,89850 & 0,90119 & 269 & 0,30 & 0,94584 & 0,94812 & 228 & 0,24 \\
\hline $40-44$ & 0,87772 & 0,88143 & 371 & 0,42 & 0,93877 & 0,94249 & 372 & 0,40 \\
\hline $45-49$ & 0,85373 & 0,85922 & 549 & 0,64 & 0,92647 & 0,93412 & 765 & 0,83 \\
\hline $50-54$ & 0,81809 & 0,82804 & 995 & 1,22 & 0,90693 & 0,92014 & 1321 & 1,46 \\
\hline $55-59$ & 0,77642 & 0,79227 & 1585 & 2,04 & 0,88224 & 0,90201 & 1977 & 2,24 \\
\hline $60-64$ & 0,71675 & 0,74102 & 2427 & 3,39 & 0,84479 & 0,87467 & 2988 & 3,54 \\
\hline 65.69 & 0,64381 & 0,67987 & 3606 & 5,60 & 0,79734 & 0,83755 & 4021 & 5,04 \\
\hline $70-74$ & 0,54456 & 0,59363 & 4907 & 9,01 & 0,72021 & 0,77064 & 5043 & 7,00 \\
\hline 75.79 & 0,41314 & 0,46987 & 5673 & 13,73 & 0,60892 & 0,67135 & 6243 & 10,25 \\
\hline 80.84 & 0,28182 & 0,33980 & 5798 & 20,57 & 0,47940 & 0,54568 & 6628 & 13,82 \\
\hline $85+$ & 0,10354 & 0,14024 & 3670 & 35,45 & 0,27383 & 0,32952 & 5569 & 20,34 \\
\hline
\end{tabular}




\begin{tabular}{|c|c|c|c|c|c|c|c|c|}
\hline \multicolumn{9}{|c|}{ Tabela 9 - Comparação entre as esperanças de vida real $\left(e_{x}\right)$ e hipotética $\left(e_{x}\right)$} \\
\hline \multirow{2}{*}{\begin{tabular}{|c|} 
Sexo \\
Idade \\
(em anos)
\end{tabular}} & \multicolumn{4}{|c|}{ Masculino } & \multicolumn{4}{|c|}{ Ferminino } \\
\hline & $e_{x}$ & $e_{x}$ & $\left|e_{x}-e_{x}\right|$ & $\frac{e_{x}-e_{x}}{e_{x}\left[\theta_{0}\right]}$ & $c_{n}$ & $e_{x}$ & $\left|e_{x}=e_{x}\right|$ & $\begin{array}{c}e_{x}-e_{x} . \\
e_{x}(x)\end{array}$ \\
\hline 0 & 65,44 & 67,04 & 1,60 & 2,44 & 73,33 & 75,24 & 1,91 & 2,61 \\
\hline 1 & 67,02 & 68,67 & 1,66 & 2,47 & 74,64 & 76,61 & 1,97 & 2,64 \\
\hline 2 & 66,28 & 67,95 & 1,66 & 2,51 & 73,92 & 75,90 & 1,98 & 2,67 \\
\hline 3 & 65,36 & 67,03 & 1,66 & 2,55 & 72,99 & 74,97 & 1,98 & 2,71 \\
\hline 4 & 64,41 & 66,07 & 1,66 & 2,58 & 72,03 & 74,00 & 1,98 & 2,74 \\
\hline $5-9$ & 63,46 & 65,11 & $1, \infty$ & 2,61 & 71,06 & 73,04 & 1,98 & 2,78 \\
\hline $10-14$ & 58,64 & 60,28 & 1,04 & 2,80 & 66,19 & 68,15 & 1,96 & 2,97 \\
\hline $15-19$ & 53,80 & 55,44 & 1,03 & 3,04 & 61,30 & 63,25 & 1,95 & 3,19 \\
\hline $20-24$ & 49,24 & 50,87 & 1,63 & 3,31 & 56,46 & 58,40 & 1,94 & 3,44 \\
\hline 25.29 & 44,82 & 46,44 & 1,62 & 3,62 & 51,64 & 53,57 & 1,93 & 3,74 \\
\hline $30-34$ & 40,48 & 42,11 & 1,62 & 4,01 & 46,82 & 48,74 & 1,92 & 4,09 \\
\hline $35-39$ & 36,23 & 37,85 & 1,62 & 4,48 & 42,07 & 43,96 & 1,89 & 4,49 \\
\hline $40-44$ & 32,03 & 33,64 & 1,62 & 5,05 & 37,37 & 39,20 & 1,84 & 4,92 \\
\hline $45-49$ & 27,86 & 29,45 & 1,59 & 5,72 & 32,83 & 34,53 & 1,70 & 5,19 \\
\hline $50-54$ & 23,96 & 25,46 & 1,50 & 6,27 & 28,48 & 30,02 & 1,54 & 5,40 \\
\hline $55-59$ & 20,11 & 21,50 & 1,39 & 6,90 & 24,21 & 25,57 & 1,36 & 5,63 \\
\hline 60.64 & 16,58 & 17,81 & 1,23 & 7,45 & 20,17 & 21,29 & 1,12 & 5,56 \\
\hline 65.69 & 13,17 & 14,19 & 1,02 & 7,72 & 16,22 & 17,13 & 0,90 & 5,57 \\
\hline 70.74 & 10,12 & 10,89 & 0,77 & 7,61 & 12,69 & 13,40 & 0,70 & 5,54 \\
\hline 75.79 & 7,54 & 8,10 & 0,56 & 7,37 & 9,56 & 10,01 & 0,45 & 4,73 \\
\hline $80-84$ & 4,89 & 5,24 & 0,35 & 7,14 & 6,46 & 6,74 & 0,28 & 4,26 \\
\hline $85+$ & 4,02 & 4,15 & 0,13 & 3,28 & 4,44 & 4,52 & 0,08 & 1,81 \\
\hline
\end{tabular}

De fato, em relação às diferenças relativas nas probabilidades de morte, tem-se que, em Fortaleza, no sexo feminino, os valores relativos foram maiores que os de São Paulo a partir da faixa de 5-9 anos, ratificando um maior impacto das neoplasias malignas, na mortalidade em Fortaleza, sobretudo em adultos jovens; nos homens, essa tendência não foi confirmada, pois nos grupos etários predomina uma cidade ou outra.

Quanto às diferenças relativas nas probabilidades de sobreviver, entre os homens, percentuais discretamente mais altos couberam à capital paulista, em todas as idades, em ambos os sexos. No que diz respeito às diferenças relativas das expectativas de vida, a capital cearense revelou números sempre levemente inferiores aos de São Paulo. ${ }^{23}$

O s resultados de Fortaleza são modestos quando confrontados com os da $\mathrm{H}$ olanda, em 1986-90, visto queo expurgo hipotético dasmortes por câncer daria um aumento da esperança de vida ao nascer de 4,0 anos (5,3\%) nos homens e de 3,4 anos $(4,2 \%)$ nas mulheres (7).
Assim, esses fatos confirmam a relevância das neoplasias malignas como problema de Saúde Pública, sobretudo tendo-se em conta que ponderável parcela dessas mortes pode ser evitada, e que, provavelmente, se comparassem tais causas com grupos de doenças redutíveis, aquelas se sobressairiam. Com efeito para Fortaleza em 1993-95, Silva ${ }^{20}$ mostrou que a exclusão de todas as causas evitáveis conferiria 5,60 e 3,32 anos aos homens e mulheres, na mesma ordem, de modo que, isoladamente, o expurgo dos cânceres proporcionaria maiores ganhos na expectativa de vida que quaisquer dos grupos de causas redutíveis, excetuando o apurado em violências nos homens.

Silva, ${ }^{25}$ analisando os anos potenciais de vida perdidos em Fortaleza (1978-80), através de técnica que Romeder e M cW hinnie, ${ }^{3}$ comprovou o peso relativo das neoplasias nos dois sexos, que participaram com 5,34\% e $10,37 \%$ das perdas potenciais masculinas e femininas, respectivamente. 
Figura 3 - Esperança de vida real $\left(e_{x}\right)$ e hipotética (e $e_{x}$ ) com eliminação das neoplasias malignas (C.I.D. 140-208) como causa de morte, segundo idade, dos residentes do município de Fortaleza, no período 1996-98 (sexo masculino)

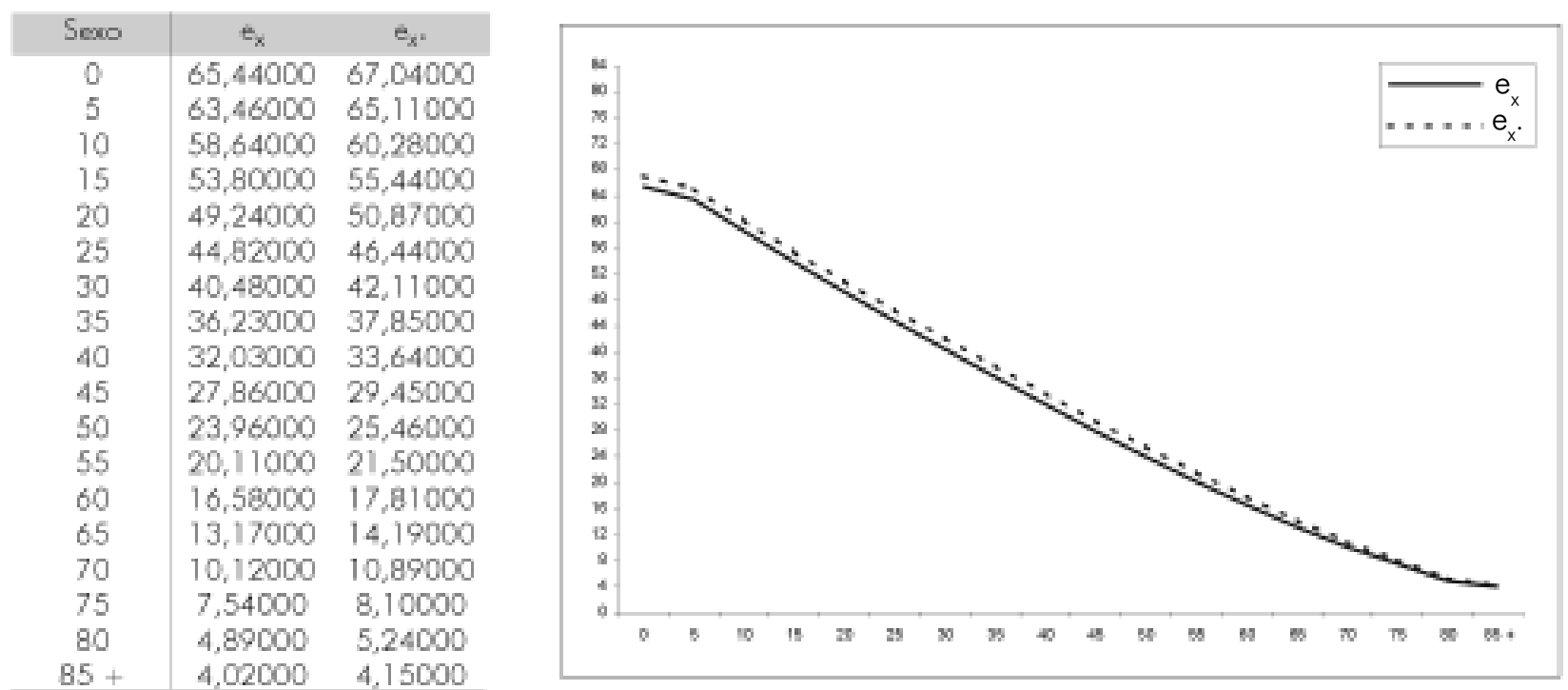

Figura 4 - Esperança de vida real $\left(e_{x}\right)$ e hipotética $\left(e_{x^{\prime}}\right)$ com eliminação das neoplasias malignas (C.I.D. 140-208) como causa de morte, segundo idade, dos residentes do município de Fortaleza, no período 1996-98 (sexo feminino)

\begin{tabular}{c|cc|}
\hline Sewo & $e_{x}$ & $e_{x^{*}}$ \\
\hline 0 & 73,33000 & 75,24000 \\
5 & 71,06000 & 73,04000 \\
10 & 66,19000 & 68,15000 \\
15 & 61,30000 & 63,25000 \\
20 & 56,46000 & 58,40000 \\
25 & 51,64000 & 53,57000 \\
30 & 46,82000 & 48,74000 \\
35 & 42,07000 & 43,96000 \\
40 & 37,37000 & 39,20000 \\
45 & 32,83000 & 34,53000 \\
50 & 28,48000 & 30,02000 \\
55 & 24,21000 & 25,57000 \\
60 & 20,17000 & 21,29000 \\
65 & 16,22000 & 17,13000 \\
70 & 12,69000 & 13,40000 \\
75 & 9,56000 & 10,01000 \\
80 & 6,46000 & 6,74000 \\
$85+$ & 4,44000 & 4,52000 \\
\hline
\end{tabular}

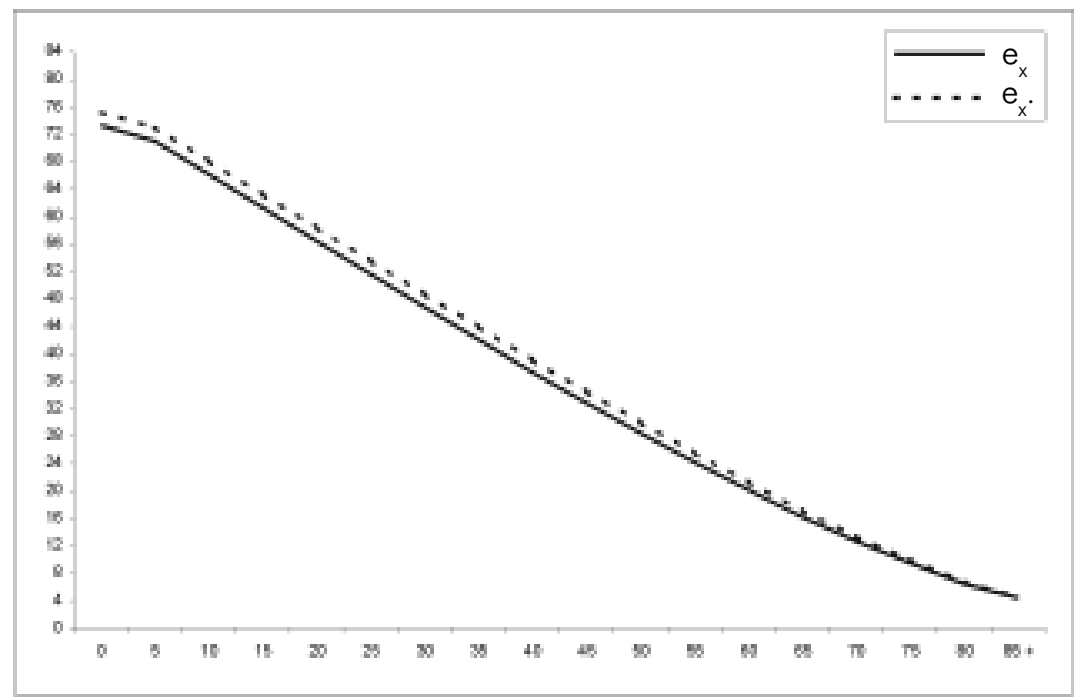

Barnum e G reeberg ${ }^{6}$ apontam que a idade média ao morrer, dos pacientes de câncer, nos países em desenvolvimento, é de 59 anos para os homens e 58 anos nas mulheres, subtraindo 20 e 21 anos de vida em média por cada morte, correspondentemente. Tal situação guarda semelhança com a de Fortaleza se aferido sob a ótica dos anos potenciais de vida perdidos.

Lai e $\mathrm{H}$ ardy ${ }^{26}$ afirmam que medir $\mathrm{O}$ impacto de risco competitivo de mortes na sociedade é importante para política de saúde pública e alocação de recursos. As tábuas de vida de múltiplo decremento (TVM D) levam em conta riscos competitivos na população e podem ser comparadas facilmente através das populações. A técnica de APVP não toma em conta riscos competitivos e é também fortemente influenciada pela estrutura etária da população e seu tamanho. Além disso, as TVMD são melhores para avaliar morte prematura de 15 a 64 anos.

É imperioso ressaltar que as neoplasias malignas, por atingirem segmentos da população economicamente ativa, mormente em mulheres, devem ter sua transcendência majorada em função das grandes repercussões sociais e econômicas delas decorrentes. Por conseguinte, devem merecer a condição de prioridade e receber uma atenção especial com intuito de atenuar os altos riscos existentes. 


\section{CONCLUSÕES}

Com base nas tábuas de vida real e hipotética pode-se concluir que:

1. a esperança de vida ao nascer foi de 65,44 anos, nos homens, e 73,33 anos, nas mulheres;

2. se as neoplasias malignas não fossem causa de morte, a probabilidade de morrer mais precocemente diminuiria e aos 85 anos chegariam $35,45 \%$ a mais de homens, e $20,34 \%$ a mais de mulheres;

3. se as neoplasias malignas não tivessem sido causa de morte da população de 15 a 65 anos, a probabilidade de sobreviver à faixa economicamente ativa aumentaria de $67,77 \%$ e $80,02 \%$ para $71,51 \%$ e $87,16 \%$, respectivamente, para os sexos masculino e feminino; e

4. se as neoplasias malignas fossem excluídas como causa de morte haveria um ganho de 1,60 anos (2,44\% a mais), e de 1,91 anos $(2,61 \%)$ nas esperanças de vida ao nascer masculina e feminina.

\section{REFERENNCIAS BIBLIOGRÁFICAS}

1. Alonso C aballero J. La medición del estado de salud: metodologia de la encuesta de sal ud. In: $\mathrm{M}$ artínez $\mathrm{N}$ avarro $\mathrm{F}$, et al. eds. Salud Pública. M adrid: M cG raw-H ill, 1998:342-61.

2. Chiang $C L$. Introduction to stochastic process in biostatistics. N ew York: J. Wiley, 1968.

3. Romeder JM, M cwhinnieJR. Ledévelopment des années potentielles de vie perdues comme indicateur de mortalité prématurée. Rev Epidemiol Sante Publique 1978;6:97-115.

4. Arriaga EE. Los años de vida perdidos: su utilización para medir el nivel y el cambio dela mortalidad. N otas Poblac 1996;63:7-38.

5. Bocco M .Lardación entrelosañosdevidaperdidosy laesperanzadevida. N otasPoblac 1996;63:39-60.

6. Barnum $\mathrm{H}, \mathrm{G}$ reeberg ER. C ancers. In: Jamison DT, M osley WH, M easham AR, BobadillaJ L, eds. D isease control in developing countries. O xford U niversity Press, 1997:529-59.

7. Bonneux L, Barendregt JJ, N usselder WJ, Van Der $M$ aasPJV. Preventing fatal diseasesincreases healthcare costs: cause elimination life table approach. Br M ed J 1998;316:26-9.

8. M errill RM, Kessler LG, U dler JM, Rasband GC, Feuer EJ. Comparison of risk estimatesfor selected diseases and causes of death. Prev M ed 1999;28:179-93.

9. Fraser GE, Shavlik D. Risk factors, lifetimerisk, and ageat on set of breast cancer. Ann Epidemiol 1997;7(6):375-82.

10. Karner-H anusch J, M ittlbck M , Fillipitsch T, $H$ erbst $F$. Family history as a marker of risk for colorectal cancer: Austrian experience. World J Surg 1997;21(2):205-9.

11. M anton KG , D owd E. M odelsfor forecasting chronic disease processes in adult and elderly populations: effects of stochasticity.J Epidemiol Biostat 1999;4(1):11-8.

12. M erril RM . M easuring the projected public health impact of lung cancer through lifetime and age-conditional risk estimates. Ann Epidemiol 2000;10(2):88-96.

13. M erril RM , Weed D L, Feuer EJ. The lifetime risk of developing prostatecancer in whiteand black men. Cancer Epidemiol Biomarkers Prev 1997;6(10):763-8.

14. N azario CM , Figueroa-Valls N, Rosario RV. B reast cancer patterns and lifetime risk of developing breast cancer among Puerto Rican females. P R H ealth Sci J 2000;19(1):7-13.

15. Wun LM , M erril RM , Feuer EJ. Estimating life timeand age conditional probabilities of developing cancer. LifetimeD ataAnal 1998;4(2):169-86.

16. Agarwal SS, M urthy NS, SharmaKC, D asDK. Evaluation of ahospital based cytology screening programmefor reduction in lifetime risk of cervical cancer. N eoplasma 1995;42(2):93-6.

17. M essori A, Trippoli S. A new method for expressing survival and lifeexpectancy in lifetime cost-efectivenessstudies that evaluate cancer pa tients(review). Oncol Rep 1999;6(5):1135-41.

18. Soutter W P. Invasive cancer after treatment of cervical intraepithelial neoplasia. Ann Acad M ed Singapore 1998;27(5):722-4.

19. Brasil. M inistério daSaúde. Instituto $\mathrm{N}$ acional de Câncer. Estimativasdaincidênciaemortalidadepor câncer no Brasil. Rio dejaneiro: IN CA, 2001. 83p.

20. Silva M GC. M ortalidade por causas evitáveis em Fortal eza de 1978 a 1995 [Tese]. Fortaleza: EscoladeSaúdePública, U niversidadeEstadual do C eará, 1998. 300p.

21. O rganização M undial da Saúde. M anual da classificação estatísticainternacional dedoenças, lesões e causas de óbitos. Revisão 1975. São Paulo, 1978. 2 vol.

22. Laurenti R, G otlieb SLD, Sousaj M P de, Lebrão M L. Estatísticas de saúde. São Paulo: EPU, EDUSP, 1985.

23. G otlieb SLD . M ortalidadediferencial por causas, São Paulo - 1970: tábuas de vida de múltiplo decremento. Rev SaúdePública 1981;15:401-17.

24. Notzon FC, KomarovYM , ErmzkovS, SemposCT, $M$ arksJS,SemposEV. Causesof dedining lifeexpectancy in Russia JAM A 1998;279(10):793-800.

25. Silva M GC. Anos potenciais devida, segundo causas, em Fortal eza, em 1978-80. Rev Saúde Pública 1984;18:108-21.

26. Lai D , H ardy RJ. Potential gainsin lifeexpectancy or years of potential lifelost: impact of competing risks of death. Int] Epidemiol 1999;28(5): 894-8. 